\title{
¿Son útiles las Escape Rooms educativas? El lado oscuro de $\pi$. Análisis del feedback inicial.
}

\author{
Lara Ferrando ${ }^{1}$ D y Lucía Rey-Lorenzo ${ }^{2}$
}

\section{Resumen}

El uso del juego matemático en el aula puede direccionar alumnado, independientemente del nivel, a mejorar sus habilidades de aprendizaje, y a la vez producir una mayor motivación e interés, por las matemáticas. Por lo tanto, a continuación, se muestran dos Escape Rooms Digitales como experiencia de enseñanza-aprendizaje para el alumnado de educación primaria y para el de educación secundaria. En este trabajo se han recopilado diferentes datos, desde las primeras impresiones de todas aquellas personas que han realizado las Escapes Rooms hasta las edades, grados de formación e incluso nacionalidad de estas. Los resultados muestran que aunque en algunos casos el nivel de la actividad haya resultado ser de grado medio, ha gustado la experiencia. Como trabajo futuro esta previsto realizar la puesta en práctica de la adaptación al aula.

\section{Abstract}

The use of mathematical play in the classroom can lead students, regardless of level, to improve their learning skills, while producing greater motivation and interest, in mathematics. Therefore, two Digital Escape Rooms are shown below as a teaching-learning experience for primary and secondary educational students. Different data have been compiled in this work, from the first impressions of all those who have made the Escape Rooms to the ages, degrees of training and even nationality of these. The results show that although in some cases the level of activity has turned out to be of medium grade, the experience has liked. As a future work it is planned to implement classroom adaptation.

\section{Palabras clave}

Gamificación educativa - Resolución de problemas - Educación Primaria - Educación Secundaria - Aprendizaje cooperativo

\section{Keywords}

Educational gamification - Problem Solving - Primary education - Secondary education - Cooperative learning

1 Universitat Jaume I

2 Universidade de Santiago de Compostela

*Autor de correspondencia: Iferrand@uji.es

\section{Introducción}

Una de las actuales y grandes dificultades con la que se encuentra el profesorado reside en la motivación de sus estudiantes. Esta falta de motivación, parece ser que viene produciéndose desde los primeros cursos de la etapa de la Educación Primaria y se alarga a cursos superiores en la Educación Secundaria. Cabe destacar que el alumnado está expuesto a múltiples estímulos tanto internos como externos, siendo la mayoría de ellos de procedencia tecnológica.

La selección de los medios más adecuados a cada situación y el diseño de buenas intervenciones en el ámbito educativo que consideren todos los elementos contextuales (contenidos, características del alumnado, circunstancias ambientales, etc.) resultan factores clave para el logro de los objetivos educativos que se pretenden con esta actividad y que son posteriormente enumerados (Olivar y Daza, 2007). Consecuentemente, a lo largo de la historia de la humanidad la educación ha sido moldeada a las necesidades sociales de cada etapa. Sin embargo, las nuevas tecnologías por sí solas no garantizan el éxito del proceso de enseñanza-aprendizaje si no hay una mentalidad de cambio por parte del profesorado. Por lo tanto, los y las docentes del nuevo siglo deben ser conscientes de la situación actual, en la que las TIC posibilitan el desarrollo de materiales más dinámicos, con interactividades y con contenidos motivadores y fáciles de manejar por el alumnado, y empezar a tomar partido en el cambio guiándolo hacia el desarrollo de las denominadas habilidades del aprendizaje del s.XXI, 
categorizadas en habilidades de información y comunicación, de pensamiento y resolución de problemas, e interpersonales y de autonomía.

Actualmente, es tanto el conocimiento que hay a nuestra disposición y a la de los y las estudiantes que se ha vuelto imposible el "conocerlo todo". Esto nos genera la necesidad de aprender cómo se relaciona lo que se conoce con lo que puede llegarse a conocer. Que el alumnado aprenda a relacionar, a encontrar y establecer conexiones en la información es un objetivo educativo en un mundo en el que se hace imprescibdible el aprender la utilización de procedimientos y metodologías que permitan establecer nuevas relaciones. Ello ha de facilitar la adaptación a unas necesidades laborales cambiantes y a una sociedad informatizada en la cual las personas tendrán que saber cómo "moverse" para extraer y elaborar conocimientos desde el flujo de información disponible (Hernández, 1996). De ahí, la presencia del trabajo globalizado en esta actividad mediante el tratamiento de contenidos histórico-geográficos y científicos.

Tal y como afirman Gómez Chacón, Figueiras Ocaña, y Marín Rodríguez (2001) "Son muchos los casos en que una pregunta interesante realizada en un plano lúdico o bien una observación ingeniosa sobre una situación aparentemente inocente han dado lugar a nuevos modos de pensar en Matemáticas." (e.g., Gómez Chacón y cols., 2001, p. 124). Estas preguntas y situaciones que son capaces de romper los bloqueos intelectuales y que hacen avanzar el pensamiento matemático surgen muy a menudo cuando somos capaces de colocarnos en una actitud distendida y juguetona, fuera del contexto serio y severo con que se reviste normalmente esta asignatura (De Guzmán, 1989).

Así pues, el juego matemático bien escogido puede conducir al alumnado, independientemente de la etapa educativa en la que se encuentre, a la mejor posición de observación y aproximación inicial a cualquiera de los temas de estudio con los que se debe de enfrentar, mejorando sus habilidades de aprendizaje gracias a la apertura, el desbloqueo, la motivación, el interés, la diversión o el entusiasmo generados (Rey-Lorenzo y Vázquez-Abal, 2020). Por lo tanto, a continuación, se plantea una Escape Room Digital como experiencia de enseñanza-aprendizaje, cuyo objetivo principal será lograr un cambio actitudinal positivo del estudiantado alejando a las matemáticas de ese halo de tedio y dificultad que las rodea mediante el empleo del recurso de gamificación educativa. Tal y como afirman Diego y Ventura-Campos (2017): La gamificación educativa "hace referencia al uso de elementos propios del juego (en el sentido tradicional) en situaciones no lúdicas, como por ejemplo en un negocio o en un aula." (e.g., Diego y Ventura-Campos, 2017, p. 34).

Por otro lado, la educación, especialmente en niveles obligatorios, debe favorecer a la formación integral del alumnado, lo que incluye el desarrollo de competencias cognitivas, y también, de competencias socio-emocionales.
A lo largo del siglo XX en EEUU surge una alternativa a la concepción educativa predominante, puesto que en esta se enfatizaba el individualismo, la memorización, la competición, la búsqueda de objetividad, etc., dando lugar a un alumnado con un desarrollo pobre en habilidades sociales de intercambio y negociación, y con un pensamiento poco reflexivo y analítico (López Haro, 2013).

Se ha demostrado que las personas que trabajan (aprenden) juntas, se implican más activamente en el proceso de aprendizaje puesto que las técnicas de aprendizaje en grupo les permiten actuar sobre su propio proceso, involucrándose más con la materia de estudio y con sus compañeros y compañeras (Gavilán, 2009). Así pues, no sorprende que este actividad que a continuación se analiza, se basase en el trabajo en equipo, el cual se caracteriza, según Domingo (2008), por la comunicación fluida entre las personas en función de las relaciones de confianza y soporte mutuo, y en el que es imprescindible la existencia de roles dinámicos.

En este artículo mostramos una propuesta con la que podremos gamificar el aula con la finalidad de motivar al alumnado mediante el juego. Nuestra actividad es una Escape Room (habitación de Escape) dividida en dos niveles, donde el primero va destinado al alumnado de primaria y el segundo, al de secundaria. Así pues, el objetivo principal de este artículo es analizar el feedback recibido por los y las participantes del juego.

\section{Detalles de la propuesta}

En el primer nivel de la Escape Room, orientado al alumnado de primaria, se pretende que las y los participantes hagan un viaje por varias comunidades autónomas de España con el fin de recoger las piezas de un ordenador para después montarlo. Dichas piezas han sido distribuidas por diferentes partes del país por un villano que está enfadado con la sociedad por el mal uso que se le da tanto a las redes sociales como a los medios de transporte, entre otros. Durante este viaje los más pequeños/as ayudarán a la Señora $\mathrm{Pi}$ en el proceso de recopilación y montaje del ordenador demostrando así su interés en salvar el planeta con todo lo que eso conlleva.

Sin embargo, el peligro de destrucción continúa, en el nivel para el alumnado de secundaría (se realiza en dos partes), se pretende que las personas participantes se sumerjan en un viaje matemático por España motivado por la búsqueda de un villano al que deberán detener, pues este, tras observar el mal uso que se le dan a las herramientas tecnológicas, pretende terminar con todas ellas a nivel mundial. Dicho individuo ha elaborado el plan desde un ordenador cuántico único en el mundo, el modelo 314-Pi, que ha desmontado y repartido sus piezas por toda España.

\subsection{Objetivos}

Los principales objetivos de ambas Escapes se centraron en: 
- Conseguir que el alumnado alcance una motivación intrínseca, de competencia y de control de logro sana.

- Trabajar en equipo reforzando las componentes de interacción promocional cara a cara, valoración personal y el auto-análisis de grupo.

- Trabajar de manera globalizada relancionando los distintos contenidos curriculares.

- Mejorar las habilidades socio-emocionales del alumnado.

- Aumentar el rendimiento académico y el grado de significatividad del aprendizaje.

- Visibilizar tanto la presencia como la utilidad de las matemáticas en contextos no académicos.

\subsection{Contenidos}

Los contenidos tratados en la Escape Room para primaria son:

- Resolución de problemas.

- Conocimiento de las comunidades autónomas y provincias.

- Comprensión auditiva.

- Cálculo operacional: adición, sustracción, multiplicación y división.

- Lenguaje de signos.

- Pensamiento lógico-matemático.

- Habilidades viso-espaciales.

- Comparación números (mayor que, menor que) y paridad.

Los contenidos tratados en la Escape Room para secundaria son:

- Pensamiento lógico-matemático.

- Sistemas de numeración.

- Radicales. Operaciones.

- Ecuaciones.

- Cuerpos y figuras geométricas. Elementos y propiedades.

- Semejanza y proporcionalidad.

- Identificación y análisis de las propiedades de las curvas.

- Derivación.

- Integración.

Además, en ambos casos se trabajarán de manera transversal otros contenidos relacionados con asignaturas como la de historia y geografía, lengua y literatura o biología.

\subsection{Enlaces a las Escape Rooms}

A continuación se muestran los links de ambas Escapes, donde se encuentran ambas en catalán, galego y castellano. Además, el nivel de secundaria también puede encontrarse en euskera.

- El lado oscuro de $\pi$ (primaria).

- El lado oscuro de $\pi$ (secundaria).

\section{Metodologia}

Ambos niveles de la Escape Room, se han realizado en formato online usando la plataforma gratuita Wix. Al final de cada Escape Room hay un enlace a una encuesta de Google Drive donde se han recopilado los datos de los y las participantes. Destacar que el enlace a las Escape Rooms se hizo público en marzo, y que hasta el momento han contestado un total de 204 personas a la encuesta.

En los siguientes subapartados se muestra la encuesta que realizaron, las variables a estudiar y su finalidad.

\subsection{Encuesta}

1. ¿A qué Escape Room has jugado? Marca todas aquellas a las que hayas jugado.

- El lado oscuro de $\pi$ Parte 1.

- El lado oscuro de $\pi$ Parte 2.

- Primaria.

2. ¿Has jugado individualmente o en grupo?

- Individualmente.

- En grupo.

3. ¿Cuántos años tienes (si es un grupo, la media de edad)?

- 0-5 años

- 6-12 años

- 13-18 años

- 19-39 años

- 40-69 años

- Más de 70.

4. ¡Queremos ver lo lejos que viaja esto! ¿De qué país eres (si eres de España, por favor, indica a qué comunidad autónoma perteneces)?

- Andalucía

- Aragón

- Asturias

- Islas Baleares

- Islas Canarias

- Cantabria 
- Castilla - La Mancha

- Castilla y León

- Cataluña

- Comunidad Valenciana

- Extremadura

- Galicia

- La Rioja

- Madrid

- Murcia

- Navarra

- País Vasco

- Ceuta

- Melilla

5. ¿Qué formación matemática tienes?

- Ninguna

- Nivel básico (primaria).

- Nivel medio (secundaria y bachillerato).

- Nivel alto (estudios universitarios afines a las matemáticas: ingeniería, física, química...).

- Grado en Matemáticas o superiores.

6. Señala el grado de dificultad del juego:

- Fácil

- Medio

- Difícil

7. Si tienes algún comentario sobre la Escape Room, escríbelo a continuación.

\subsection{Variables} fueron:

Las variables que se estudiaron a partir de la encuesta

- Nivel: Indica el nivel de la Escape Room que habían realizado los y las participantes.

- Modalidad: Indica la modalidad del juego, si se ha jugado de forma individual o grupal.

- Edad: Indica la edad de cada participante o, en el caso de haber jugado en grupo, la media aritmética de las edades.

- Lugar: Indica el lugar de procedencia de los y las participantes.

- Formación: Indica el nivel académico de los y las participantes

- Dificultad: Indica el grado de dificultad que supuso a los y las participantes al realizar la Escape Room.

\section{Resultados}

\subsection{Variable: Nivel}

Después de un par de meses desde el lanzamiento de las Escape Rooms, un total de 204 personas han contestado a la encuesta voluntaria final. Como puede observarse en la figura 1 , tan solo un 3,4\% ha realizado las tres actividades. Además, el porcentaje de personas que han completado las dos partes de la Escape Room de secundaria es mayor que el porcentaje de aquellas que solamente han hecho una de las partes (el $33,3 \%)$.

¿A qué Escape Room has jugado? Marca todas aquellas a las que hayas jugado.
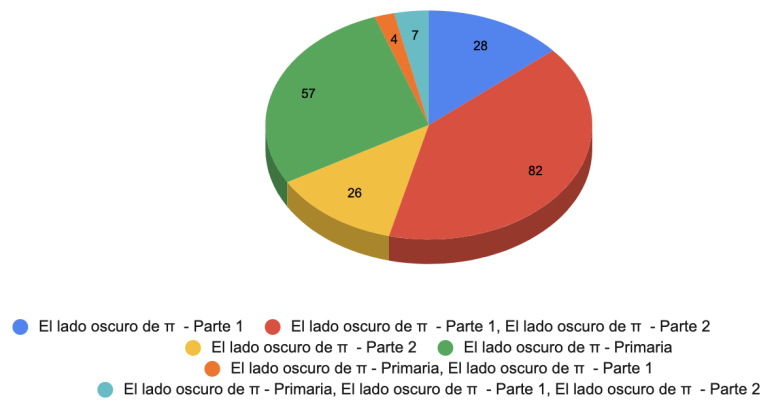

Figura 1. Gráfico donde se expresan los porcentajes y número de personas que han jugado a cada una de las Escape Room. El lado oscuro de $\pi$ Parte 1/ Parte 2 hace referencia al nivel de secundaria.

\subsection{Variable: Modalidad}

En el siguiente diagrama de barras (figura 2) puede observarse que más de la mitad de los participantes han jugado de forma grupal, donde 78 lo han hecho de forma individual.

\section{¿Has jugado individualmente o en grupo?}

150

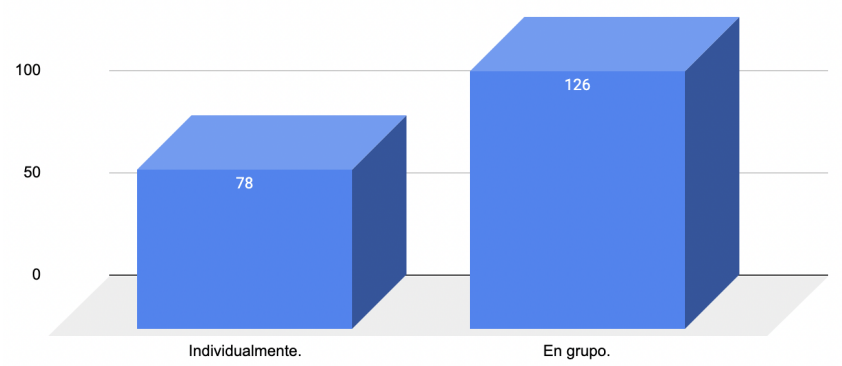

Figura 2. Diagrama de barras donde donde aparece la modalidad de juego general.

\subsection{Variable: Lugar}

La otra variable a analizar nos indicaba desde donde se estaba realizando la actividad. Tal y como puede observar en la figura 3, gran parte de los y las participantes provienen de Andalucía. Además, se observa que la actividad ha sido realizada fuera del territorio español. 
¡Queremos ver lo lejos que viaja esto! ¿De qué país eres (si eres de España, por favor, indica a qué comunidad autónoma
perteneces)?

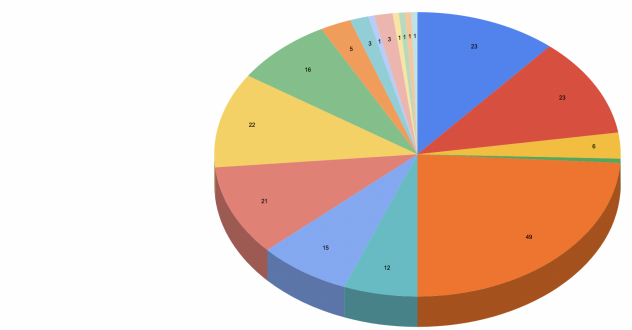

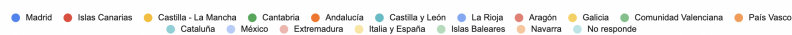

Figura 3. Gráfico donde se muestran las comunidades españolas y países desde donde se ha realizado la actividad.

\subsection{Variable: Edad}

En la figura 4 vemos que justo el $50 \%$ de los y las participantes tenían entre 13 y 18 años, seguido por el $30,4 \%$ que corresponde a la franja de edad entre los 6 y los 12 años.

\section{¿Cuántos años tienes?}

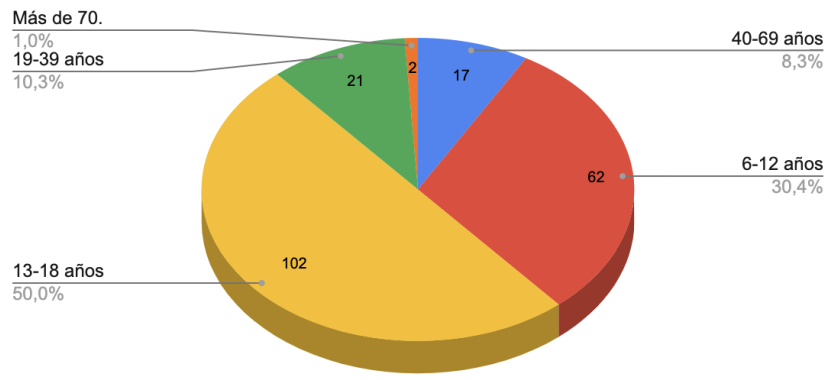

Figura 4. Gráfico donde se muestran las edades sin diferenciar niveles.

Para ver en detalle las edades de los y las participantes según el nivel de la Escape Room que han realizado, se adjuntan a continuación las figuras 5, 6 y 7 donde se detalla la información.

De aquellas personas que solamente han jugado al nivel de primaria, el $75,4 \%$ se encuentran en el rango de edad de 6 a 12 años, correspondiente al ciclo de primaria o al primer curso de secundaria.

\section{¿Cuántos años tienes? Escape Room Primaria}

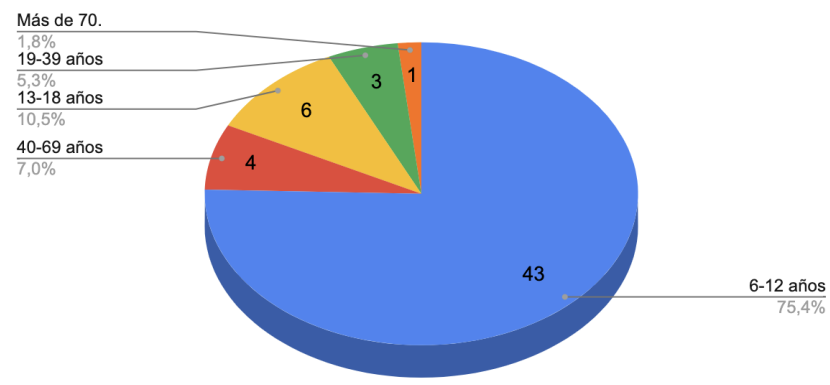

Figura 5. Gráfico donde se muestran las edades de aquellas personas que han realizado la Escape Room de primaria.
En el caso de aquellas personas que han realizado la Escape Room de secundaria, se observa que el $67,6 \%$ pertenecen al rango de edad entre 13 y 18 años, que se correspondería con el alumnado de educación secundaria y bachillerato.

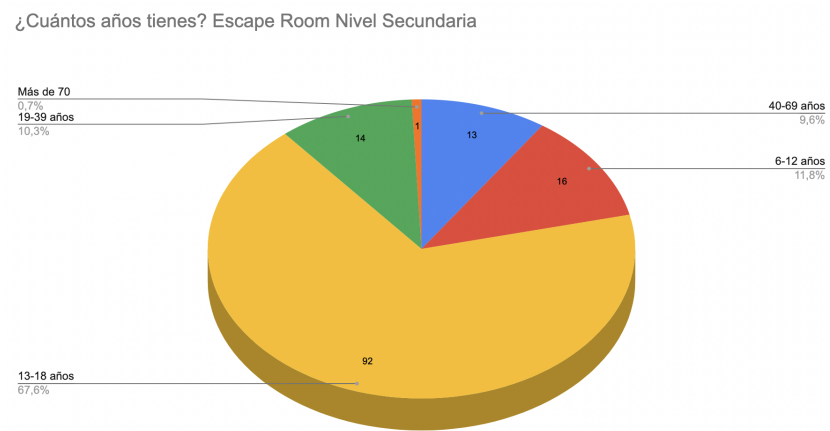

Figura 6. Gráfico donde se muestran las edades de aquellas personas que han realizado uno o ambos niveles de la Escape Room de secundaria pero no la de primaria.

Por último en este tercer gráfico, vemos que de las 7 personas que han realizado todos los niveles de la Escape Room, dos pertenecen a la franja de 6 a 12 años (franja que engloba primer curso de secundaria), cuatro a la franja de 13 a 18 años y solamente una persona que se encuentra en el rango de 19 a 39 años. Destacar que el gráfico que falta para sumar el total de edades corresponde a los y las participantes que solamente han realizado el primer nivel de secundaria y la de primaria, al ser solamente cuatro personas, se ha obviado el gráfico.

\section{¿Cuántos años tienes? Las tres Escape Room}

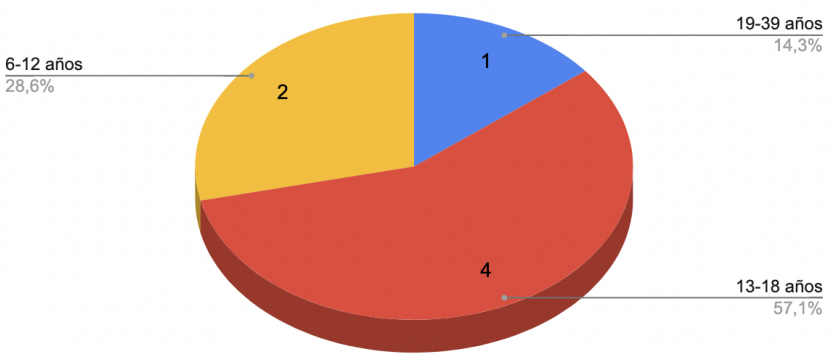

Figura 7. Gráfico donde se muestran las edades de aquellas personas que han realizado tanto los dos niveles de la Escape Room de secundaria como el nivel de primaria.

\subsection{Variable: Formación}

La siguiente variable a analizar ha sido la formación matemática que tenían las y los participantes. Los resultados pueden verse en la figura 8 , donde se observa que la mayoría de los y las participantes tenían un nivel medio que se corresponde con la etapa de secundaria y bachillerato, seguido por el 25,5\% que asociado al nivel básico. Destacar que esta gráfica contiene la formación de todas las personas que han realizado o bien un único nivel de la Escape Room, dos o los tres niveles. 

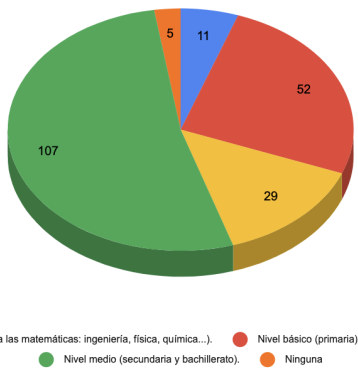

Figura 8. Gráfico donde se muestran la formación de aquellas personas que han realizado tanto los dos niveles de la Escape Room de secundaria como el nivel de primaria.

\subsection{Variable: Dificultad}

Ya por último, la variable analizada ha sido el grado de dificultad que ha supuesto la Escape Room para los y las participantes. En este caso veremos los resultados para aquellas personas que solamente han realizado el nivel de primaria (figura 9), la primera parte de la de secundaria (figura 10), la segunda parte de secundaria (figura 11) y la de secundaria completa (figura 12). El grado de dificultad para aquellas personas que realizaban las tres Escape Rooms o que hacían la de primaria y una parte de secundaria, no se mostrará ya que sería una respuesta conjunta que contemplaría dos grados diferentes (nivel primaria y los dos de secundaria).

En el gráfico de la figura 9, puede observarse que a más de la mitad de las personas la Escape Room les ha resultado fácil, así pues un $31,6 \%$ opina que la dificultad era media, y por último una única persona asocia el grado de dificultad como difícil.

\section{Señala el grado de dificultad del juego Primaria:}

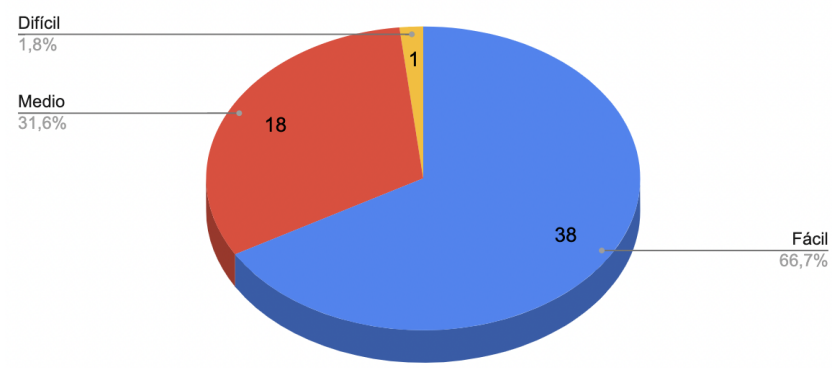

Figura 9. Gráfico donde se muestra el grado de dificultad experimentado por las personas que han realizado el nivel de primaria.

Ahora, si comparamos las figuras 10 y 11, podemos ver que para aquellos que solamente han realizado la parte 1 el porcentaje que se asocia al grado de dificultad más alto difiere en más de un $14,1 \%$ con el de la parte 2 . No obstante, en ambos casos determina que el grado de dificultad es medio.
Señala el grado de dificultad del juego Parte 1:

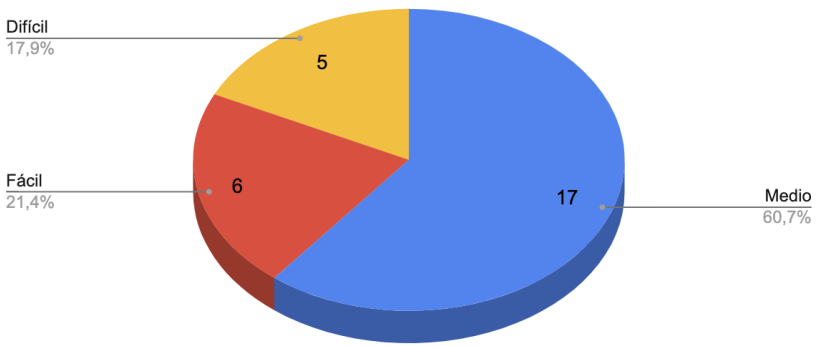

Figura 10. Gráfico donde se muestra el grado de dificultad experimentado por aquellas personas que han realizado únicamente la primera parte de la Escape Room de secundaria.

Señala el grado de dificultad del juego Parte 2:

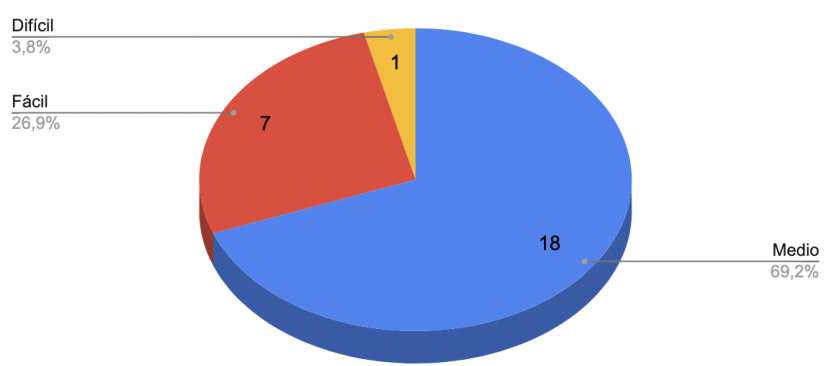

Figura 11. Gráfico donde se muestra el grado de dificultad experimentado por aquellas personas que han realizado únicamente la segunda parte de la Escape Room de secundaria.

A continuación veremos los resultados obtenidos de aquellas personas que han realizado ambas partes y que han hecho una valoración global (figura 12). Más de tres cuartas partes de todas las personas que han realizados la Escape Room de secundaria completa, le asignan una dificultad media. A este le sigue el de dificultad alta (grado difícil), siendo así el menor porcentaje el de dificultad baja (fácil).

\subsection{Gusto por el juego:}

Uno de los apartados del cuestionario recogía las opiniones respecto a la Escape Room. En general, mostraron haber ganado interés en resolver los problemas, apuntando también qué partes del juego les habían resultado más difíciles. Además, en varias ocasiones se ha afirmado que aquellas personas que estaban realizando la Escape Room eran docentes, y que próximamente llevarían la actividad a su aula.

\section{Discusión y trabajo futuro}

El presente trabajo tenía como objetivo analizar el feedback recibido por los y las participantes de las Escape Rooms, dos meses después del lanzamiento. Es por ello que podemos afirmar que se ha cumplido. A través de las respuestas de los 204 encuestados y encuestadas se ha podido observar que la 
Señala el grado de dificultad del juego Parte 1 y 2 :

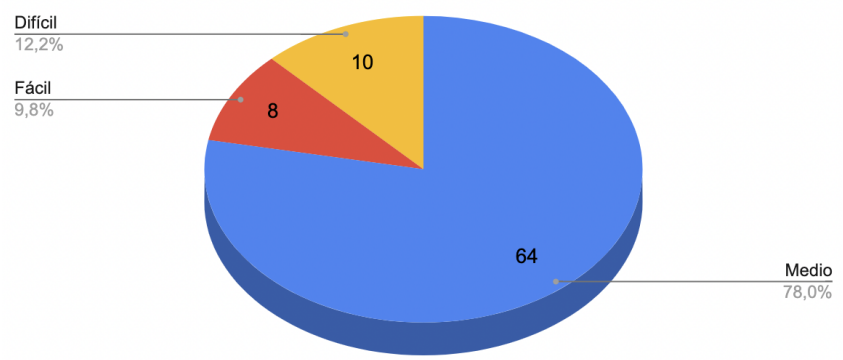

Figura 12. Gráfico donde se muestran muestra el grado de dificultad experimentado por aquellas personas que han realizado las dos partes de la Escape Room de secundaria.

actividad se ha realizado en más casos de forma grupal, contribuyendo así al trabajo cooperativo entre los y las participantes. En cuanto a las edades, se ha visto que no solamente una franja de edad ha realizado uno de los niveles de la Escape Room sino que en todos los niveles hay variedad en el rango de estas. Es por ello que quizás, en general haya resultado fácil el nivel de primaria, debido a la variedad de edades, y por lo consecuente que el de secundaria resulte de dificultad media. Además, como era previsto, se encuentra una relación entre las edades y el grado de formación, puesto que hay tantos participantes del nivel de secundaria como personas que han jugado pertenecientes al rango de edad correspondiente a esta etapa. Ya por último, comentar que en general el juego ha gustado a los y las participantes, puesto que se ha comentado que es una forma diferente de aprender matemáticas, geografía e historia.

Como trabajo futuro está previsto realizar de forma inminente una adaptación al aula de la Escape Room virtual de primaria para poder ser puesta en práctica en la misma de manera manipulativa y prescindiendo de los medios digitales.

\section{Referencias}

De Guzmán, M. (1989). Juegos y matemáticas (n. ${ }^{\circ}$ 4). Suma: Revista sobre Enseñanza y Aprendizaje de las Matemáticas.

Diego, P., y Ventura-Campos, N. (2017). Escape Room: gamificación educativa para el aprendizaje de las matemáticas (Vol. 85).

Domingo, J. (2008). El aprendizaje cooperativo (n. $\left.{ }^{0} 21\right)$. Cuaderno de trabajo social.

Gavilán, P. (2009). Aprendizaje cooperativo. papel del conflicto sociocognitivo en el desarrollo intelectual. consecuencias pedagógicas. Revista española de pedagogía, 87(242).

Gómez Chacón, I. M., Figueiras Ocaña, L., y Marín Rodríguez, M. (2001). Matemáticas en la red: Internet en el aula de Secundaria. Madrid: Narcea.
Hernández, F. (1996). La globalización mediante proyectos de trabajo (n. $\left.{ }^{\circ} 155\right)$. Cuadernos de pedagogía.

López Haro, I. (2013). Aprendizaje cooperativo con actividades motivadoras en matemáticas. Máster en profesorado de Educación Secundaria. Universidad de Almería.

Olivar, A., y Daza, A. (2007). Las tecnologías de la información y comunicación (tic) y su impacto en la educación del siglo xxi. Revista Negotium, 3(7).

Rey-Lorenzo, L., y Vázquez-Abal, E. (2020). Escapando de las matemáticas (n. ${ }^{\circ}$ 104). Localización: Epsilon: Revista de la Sociedad Andaluza de Educación Matemática 'Thales'. 